\title{
Optimal Control with Time Delays via the Penalty Method
}

\author{
Mohammed Benharrat ${ }^{1}$ and Delfim F. M. Torres ${ }^{2}$ \\ ${ }^{1}$ Département de Mathématiques et Informatique, Ecole National Polytechnique d'Oran (Ex. ENSET d'Oran), \\ 1523 El M'Naouar, Oran, Algeria \\ ${ }^{2}$ Department of Mathematics, Center for Research and Development in Mathematics and Applications (CIDMA), \\ University of Aveiro, 3810-193 Aveiro, Portugal
}

Correspondence should be addressed to Delfim F. M. Torres; delfim@ua.pt

Received 3 June 2014; Accepted 19 July 2014; Published 2 September 2014

Academic Editor: Ligang Wu

Copyright (C) 2014 M. Benharrat and D. F. M. Torres. This is an open access article distributed under the Creative Commons Attribution License, which permits unrestricted use, distribution, and reproduction in any medium, provided the original work is properly cited.

\begin{abstract}
We prove necessary optimality conditions of Euler-Lagrange type for a problem of the calculus of variations with time delays, where the delay in the unknown function is different from the delay in its derivative. Then, a more general optimal control problem with time delays is considered. Main result gives a convergence theorem, allowing us to obtain a solution to the delayed optimal control problem by considering a sequence of delayed problems of the calculus of variations.
\end{abstract}

\section{Introduction}

Over the past years, there has been an increasing interest in time delay problems of the calculus of variations and control [1-4]. Such interest is explained for their importance in control and engineering [5-8]. Indeed, time delays are inherent in various real systems, such as control systems and optimal control problems in engineering $[9,10]$.

In this paper we improve recent optimality conditions for time delay variational problems. In [11] necessary optimality conditions of Euler-Lagrange, DuBois-Reymond, and Noether type were obtained for problems of the calculus of variations with a time delay. The results of [11] were then extended to delayed variational problems with higher order derivatives in [2]. Here we model time delay variational problems in a more realistic way: while in $[2,11]$ the delay on functions and their derivatives (and control variables) is always the same, here we consider different delays for the functions and derivatives/controls.

The text is organized as follows. In Section 2 we formulate the delayed problem of the calculus of variations, where the delay in the unknown functions is different from the delay in their derivatives. The main result in this section is Theorem 4, which provides necessary optimality conditions of Euler-Lagrange type. Control strategies via an exterior penalty method are then investigated in Section 3 . The idea is to replace the optimal control problem with time delays by a series of delayed problems of the calculus of variations. The main result gives a convergence theorem that allows us to obtain a solution to delayed optimal control problems with linear delayed control systems, by considering a sequence of variational problems with time delays of the type considered before in Section 2 (see Theorem 7). We end with Section 4 of conclusions.

\section{Calculus of Variations with Time Delays}

We consider the following fundamental problem of the calculus of variations with time delays, where the delay in the function we are looking for is different from the delay in its derivative:

$$
\min \int_{0}^{T} L\left(t, x(t), x\left(t-\tau_{1}\right), \dot{x}(t), \dot{x}\left(t-\tau_{2}\right)\right) d t
$$

subject to

$$
\begin{gathered}
x(t)=\theta_{1}(t), \quad t \in\left[-\tau_{1},-\tau_{2}\right]=: I_{1}, \\
x(t)=\theta_{2}(t), \quad t \in\left[-\tau_{2}, 0\right]=: I_{2}, \\
x(T)=\alpha,
\end{gathered}
$$


where $L:[0, \top] \times \mathbb{R}^{4 N} \rightarrow \mathbb{R},(t, a, \bar{a}, b, \bar{b}) \rightarrow L(t, a, \bar{a}, b, \bar{b})$, is the Lagrangian, $T>0$ is fixed in $\mathbb{R}, \tau_{1}$ and $\tau_{2}$ are two given positive real numbers such that $\tau_{2}<\tau_{1}<\mathrm{T}$, and $\theta_{1}(\cdot)$ and $\theta_{2}(\cdot)$ are given piecewise smooth functions. Let $I:=[0, \top]$, $L^{2}\left(I, \mathbb{R}^{N}\right)$ be the Lebesgue space of measurable functions such that

$$
\|x\|_{L^{2}}=\left(\int_{0}^{T}\|x(t)\|_{\mathbb{R}^{N}}^{2} d t\right)^{1 / 2}<\infty
$$

and $H^{1}\left(I, \mathbb{R}^{N}\right)$ the Sobolev space of functions having their weak first derivative lying in $L^{2}\left(I, \mathbb{R}^{N}\right)$ and represented by

$$
x(t)=x(\tau)+\int_{\tau}^{t} \dot{x}(s) d s
$$

for all $\tau$ and $t$ in $I$. We denote that

(i) $\mathscr{H}$ is the space of all functions $x:\left[-\tau_{1}, \top\right] \rightarrow \mathbb{R}^{N}$ such that $x_{/ I_{1}} \in L^{2}\left(I_{1}, \mathbb{R}^{N}\right), x_{/ I_{2}} \in H^{1}\left(I_{2}, \mathbb{R}^{N}\right)$, and $x_{/ I} \in H^{1}\left(I, \mathbb{R}^{N}\right)$, which is a Hilbert space with the norm

$$
\|x\|_{\mathscr{H}}=\left(\left\|x_{/ I_{1}}\right\|_{L^{2}\left(I_{1}, \mathbb{R}^{N}\right)}^{2}+\left\|x_{/ I_{2}}\right\|_{H^{1}\left(I_{2}, \mathbb{R}^{N}\right)}^{2}+\left\|x_{/ I}\right\|_{H^{1}\left(I, \mathbb{R}^{N}\right)}^{2}\right)^{1 / 2}
$$

(ii) $D:=\left\{x(\cdot) \in \mathscr{H}: x_{/ I_{1}}=\theta_{1}, x_{/ I_{2}}=\theta_{2}\right.$, and $\left.x(\top)=\alpha\right\}$;

(iii) $J: \mathscr{H} \rightarrow \mathbb{R}$ is the functional

$$
J(x(\cdot))=\int_{0}^{\top} L\left(t, x(t), x\left(t-\tau_{1}\right), \dot{x}(t), \dot{x}\left(t-\tau_{2}\right)\right) d t .
$$

Our problem (1) and (2) takes then the following form:

$$
\min _{x(\cdot) \in D} J(x(\cdot)) .
$$

We make the following assumptions on the data of problem (7):

$\left(A_{1}\right)$ Lagrangian $L$ is a $C^{1}$ Carathéodory mapping; that is, it is of class $C^{1}$ in $(a, \bar{a}, b, \bar{b})$ for almost all $t \in[0, \top]$ and is measurable in $t$ for every $(a, \bar{a}, b, \bar{b})$;

$\left(A_{2}\right)$ there exist $\gamma_{i}(\cdot) \in L^{2}\left(I, \mathbb{R}^{+}\right), i=1, \ldots, 5$, such that a.e. in $t \in I$

$$
\begin{gathered}
|L(t, a, \bar{a}, b, \bar{b})| \leq \gamma_{1}(t), \\
\left\|\partial_{i} L(t, a, \bar{a}, b, \bar{b})\right\| \leq \gamma_{i}(t), \quad i=2, \ldots, 5,
\end{gathered}
$$

where $\partial_{i} L$ is the partial derivative of $L$ with respect to its $i$ th argument.

Definition 1 (cone of tangents). Let $Z$ be a normed space, $A \subset Z$, and $a \in \bar{A}$. The cone of tangents $T(A, a)$ is the set of all $z \in Z$ with the property that there is a sequence $\left(a_{n}\right)$ in $A$ converging strongly to $a$ and a sequence of nonnegative numbers $\left(\alpha_{n}\right)$ such that $\alpha_{n}\left(a_{n}-a\right) \rightarrow z$.
Lemma 2. The set $D$ is an affine linear subspace of $\mathscr{H}$ and the cone of tangents $T(D, x(\cdot))$ is given by

$$
\begin{aligned}
T & (D, x(\cdot)) \\
& =\left\{v(\cdot) \in \mathscr{H}: v(\cdot)_{/ I_{1}}=0, v(\cdot)_{/ I_{2}}=0, v(T)=0\right\} .
\end{aligned}
$$

Proof. Let $v(\cdot) \in T(D, x(\cdot))$. Then there exist $\left(x_{n}(\cdot)\right)_{n} \subset D$ and $\lambda_{n} \geq 0$ such that $x_{n}(\cdot) \rightarrow x(\cdot)$ in $D$ implies that $\lambda_{n}\left(x_{n}(\cdot)-\right.$ $x(\cdot)) \rightarrow v(\cdot)$ in $\mathscr{H}$. Since $x_{n}(\cdot), x(\cdot) \in D$ for all $n$, we have

$$
\begin{gathered}
x_{n}(\tau)=x(\tau)=\theta_{1}(\tau), \quad \tau \in\left[-\tau_{1},-\tau_{2}\right], \\
x_{n}(\tau)=x(\tau)=\theta_{2}(\tau), \quad \tau \in\left[-\tau_{2}, 0\right], \\
x_{n}(\top)=x(\top)=\alpha .
\end{gathered}
$$

Hence,

$$
\begin{gathered}
\lambda_{n}\left(x_{n}(\tau)-x(\tau)\right)=0, \quad \tau \in\left[-\tau_{1},-\tau_{2}\right], \\
\lambda_{n}\left(x_{n}(\tau)-x(\tau)\right)=0, \quad \tau \in\left[-\tau_{2}, 0\right], \\
\lambda_{n}\left(x_{n}(\top)-x(\top)\right)=0 .
\end{gathered}
$$

Therefore, $v(\cdot) \in \mathscr{H}$ with $v(\tau)=0$ for all $\tau \in\left[-\tau_{1},-\tau_{2}\right], v(\tau)=$ 0 for almost all $\tau \in\left[-\tau_{2}, 0\right]$, and $v(T)=0$. Thus,

$$
\begin{aligned}
T(D, x(\cdot)) & \\
\subset & \left\{v(\cdot) \in \mathscr{H}: v(\cdot)_{/ I_{1}}=0, v(\cdot)_{/ I_{2}}=0, v(\mathrm{~T})=\alpha\right\} \\
& =K .
\end{aligned}
$$

Conversely, let $v(\cdot) \in K$ for $x(\cdot) \in D$. Define $x_{n}(\cdot)=x(\cdot)+$ $(1 / n) v(\cdot)$. Then $n\left(x_{n}(\cdot)-x(\cdot)\right)=v(\cdot)$ with $v(\cdot) \in \mathscr{H}$. Hence, $v(\cdot) \in T(D, x(\cdot))$. by

For convenience, we introduce the operator $[\cdot]_{\tau_{1}}^{\tau_{2}}$ defined

$$
[x]_{\tau_{1}}^{\tau_{2}}(t)=\left(t, x(t), x\left(t-\tau_{1}\right), \dot{x}(t), \dot{x}\left(t-\tau_{2}\right)\right) .
$$

Proposition 3. Under conditions $\left(A_{1}\right)$ and $\left(A_{2}\right)$, the mapping $J(\cdot)$ is Fréchet differentiable and

$$
\begin{aligned}
J^{\prime}(x(\cdot))(v(\cdot))=\int_{0}^{\top} & \left(\left\langle\partial_{2} L[x]_{\tau_{1}}^{\tau_{2}}(t), v(t)\right\rangle\right. \\
& +\left\langle\partial_{3} L[x]_{\tau_{1}}^{\tau_{2}}(t), v\left(t-\tau_{1}\right)\right\rangle \\
& +\left\langle\partial_{4} L[x]_{\tau_{1}}^{\tau_{2}}(t), \dot{v}(t)\right\rangle \\
& \left.+\left\langle\partial_{5} L[x]_{\tau_{1}}^{\tau_{2}}(t), \dot{v}\left(t-\tau_{2}\right)\right\rangle\right) d t .
\end{aligned}
$$

Proof. Let $v(\cdot) \in \mathscr{H}$. We have

$$
\begin{aligned}
J^{\prime}(x(\cdot) ; v(\cdot)) & =\lim _{\lambda \rightarrow 0^{+}} \frac{1}{\lambda}(J(x(\cdot)+\lambda v(\cdot))-J(x(\cdot))) \\
& =\lim _{\lambda \rightarrow 0^{+}} \int_{0}^{\top} \frac{1}{\lambda}\left[L[x+\lambda v]_{\tau_{1}}^{\tau_{2}}(t)-L[x]_{\tau_{1}}^{\tau_{2}}(t)\right] d t .
\end{aligned}
$$


Define

$$
\Psi_{\lambda}(t)=\frac{1}{\lambda}\left[L[x+\lambda v]_{\tau_{1}}^{\tau_{2}}(t)-L[x]_{\tau_{1}}^{\tau_{2}}(t)\right]
$$

and

$$
\begin{aligned}
\Psi(t)= & \left\langle\partial_{2} L[x]_{\tau_{1}}^{\tau_{2}}(t), v(t)\right\rangle \\
& +\left\langle\partial_{3} L[x]_{\tau_{1}}^{\tau_{2}}(t), v\left(t-\tau_{1}\right)\right\rangle \\
& +\left\langle\partial_{4} L[x]_{\tau_{1}}^{\tau_{2}}(t), \dot{v}(t)\right\rangle \\
& +\left\langle\partial_{5} L[x]_{\tau_{1}}^{\tau_{2}}(t), \dot{v}\left(t-\tau_{2}\right)\right\rangle .
\end{aligned}
$$

Then, $\Psi_{\lambda}(t) \rightarrow \Psi(t)$ as $\lambda \rightarrow 0^{+}$for almost all $t \in[0, T]$. On the other hand,

$$
\left|\Psi_{\lambda}(t)\right| \leq g(t)
$$

a.e. in $t \in[0, T]$ with

$$
\begin{aligned}
g(t)=\gamma(t)\left[\|v(t)\|_{\mathbb{R}^{N}}+\left\|v\left(t-\tau_{1}\right)\right\|_{\mathbb{R}^{N}}\right. \\
\left.+\|\dot{v}(t)\|_{\mathbb{R}^{N}}+\left\|v\left(t-\tau_{2}\right)\right\|_{\mathbb{R}^{N}}\right]
\end{aligned}
$$

a function not depending on $\lambda$, and

$$
\left|\Psi_{\lambda}(t)\right| \leq g(t)+1
$$

for almost all $t \in[0, T]$ and $\lambda$ sufficiently small. Since $[0, T]$ has finite measure, Lebesgue's theorem yields that

$$
\int_{0}^{\top} \Psi_{\lambda}(t) d t \longrightarrow \int_{0}^{\top} \Psi(t) d t
$$

as $\lambda \rightarrow 0^{+}$. Hence,

$$
\begin{aligned}
J^{\prime}(x(\cdot))(v(\cdot))=\int_{0}^{T} & \left(\left\langle\partial_{2} L[x]_{\tau_{1}}^{\tau_{2}}(t), v(t)\right\rangle\right. \\
& +\left\langle\partial_{3} L[x]_{\tau_{1}}^{\tau_{2}}(t), v\left(t-\tau_{1}\right)\right\rangle \\
& +\left\langle\partial_{4} L[x]_{\tau_{1}}^{\tau_{2}}(t), \dot{v}(t)\right\rangle \\
& \left.+\left\langle\partial_{5} L[x]_{\tau_{1}}^{\tau_{2}}(t), \dot{v}\left(t-\tau_{2}\right)\right\rangle\right) d t .
\end{aligned}
$$

This is the directional derivative of $J$ in the direction $v$. To finish the proof, we need to show that $J^{\prime}(x(\cdot) ; v(\cdot))$ is linear and bounded in $v$ and continuous in $x$. The linearity is obvious. We begin by proving that $J^{\prime}(x(\cdot) ; \cdot)$ is bounded from $\mathscr{H}$ to $\mathbb{R}:$

$$
\left|J^{\prime}(x(\cdot) ; v(\cdot))\right| \leq \int_{0}^{\top}\left|\left\langle\partial_{2} L[x]_{\tau_{1}}^{\tau_{2}}(t), v(t)\right\rangle\right| d t
$$

$$
\begin{aligned}
& +\int_{0}^{\top}\left|\left\langle\partial_{3} L[x]_{\tau_{1}}^{\tau_{2}}(t), v\left(t-\tau_{1}\right)\right\rangle\right| d t \\
& +\int_{0}^{\top}\left|\left\langle\partial_{4} L[x]_{\tau_{1}}^{\tau_{2}}(t), \dot{v}(t)\right\rangle\right| d t \\
& +\int_{0}^{\top}\left|\left\langle\partial_{5} L[x]_{\tau_{1}}^{\tau_{2}}(t), \dot{v}\left(t-\tau_{2}\right)\right\rangle\right| d t \\
& \leq \int_{0}^{T} \gamma_{2}(t)\|v(t)\|_{\mathbb{R}^{N}} d t \\
& +\int_{0}^{T} \gamma_{3}(t)\left\|v\left(t-\tau_{1}\right)\right\|_{\mathbb{R}^{N}} d t \\
& +\int_{0}^{T} \gamma_{4}(t)\|\dot{v}(t)\|_{\mathbb{R}^{N}} d t \\
& +\int_{0}^{T} \gamma_{5}(t)\left\|\dot{v}\left(t-\tau_{2}\right)\right\|_{\mathbb{R}^{N}} d t \\
& \leq \int_{0}^{T} \gamma_{2}(t)\|v(t)\|_{\mathbb{R}^{N}} d t \\
& +\int_{-\tau_{1}}^{T-\tau_{1}} \gamma_{3}\left(t+\tau_{1}\right)\|v(t)\|_{\mathbb{R}^{N}} d t \\
& +\int_{0}^{T} \gamma_{4}(t)\|\dot{v}(t)\|_{\mathbb{R}^{N}} d t \\
& +\int_{-\tau_{2}}^{T-\tau_{2}} \gamma_{5}\left(t+\tau_{2}\right)\|\dot{v}(t)\|_{\mathbb{R}^{N}} d t \\
& \leq \int_{0}^{T} \gamma_{2}(t)\|v(t)\|_{\mathbb{R}^{N}} d t \\
& +\int_{-\tau_{1}}^{-\tau_{2}} \gamma_{3}\left(t+\tau_{1}\right)\|v(t)\|_{\mathbb{R}^{N}} d t \\
& +\int_{-\tau_{2}}^{0} \gamma_{3}\left(t+\tau_{1}\right)\|v(t)\|_{\mathbb{R}^{N}} d t \\
& +\int_{0}^{T-\tau_{1}} \gamma_{3}\left(t+\tau_{1}\right)\|v(t)\|_{\mathbb{R}^{N}} d t \\
& +\int_{0}^{T} \gamma_{4}(t)\|\dot{v}(t)\|_{\mathbb{R}^{N}} d t \\
& +\int_{-\tau_{2}}^{0} \gamma_{5}\left(t+\tau_{2}\right)\|\dot{v}(t)\|_{\mathbb{R}^{N}} d t \\
& \leq M\|v(\cdot)\|_{\mathscr{H}} .
\end{aligned}
$$


We still need to prove the continuity of $J^{\prime}(\cdot)$. Let $x_{n}(\cdot) \rightarrow x(\cdot)$ in $\mathscr{H}$. Then,

$$
\begin{aligned}
\left|\left[J^{\prime}\left(x_{n}(\cdot)\right)-J^{\prime}(x(\cdot))\right](v(\cdot))\right| & \\
\leq & \int_{0}^{T}\left|\left\langle\partial_{2} L\left[x_{n}-x\right]_{\tau_{1}}^{\tau_{2}}(t), v(t)\right\rangle\right| d t \\
& +\int_{0}^{T}\left|\left\langle\partial_{3} L\left[x_{n}-x\right]_{\tau_{1}}^{\tau_{2}}(t), v\left(t-\tau_{1}\right)\right\rangle\right| d t \\
& +\int_{0}^{T}\left|\left\langle\partial_{4} L\left[x_{n}-x\right]_{\tau_{1}}^{\tau_{2}}(t), \dot{v}(t)\right\rangle\right| d t \\
& +\int_{0}^{T}\left|\left\langle\partial_{5} L\left[x_{n}-x\right]_{\tau_{1}}^{\tau_{2}}(t), \dot{v}\left(t-\tau_{2}\right)\right\rangle\right| d t \\
\leq & \int_{0}^{T}\left\|\partial_{2} L\left[x_{n}-x\right]_{\tau_{1}}^{\tau_{2}}(t)\right\|\|v(t)\|_{\mathbb{R}^{N}} d t \\
& +\int_{0}^{T}\left\|\partial_{3} L\left[x_{n}-x\right]_{\tau_{1}}^{\tau_{2}}(t)\right\|\left\|v\left(t-\tau_{1}\right)\right\|_{\mathbb{R}^{N}} d t \\
& +\int_{0}^{T}\left\|\partial_{4} L\left[x_{n}-x\right]_{\tau_{1}}^{\tau_{2}}(t)\right\|\|\dot{v}(t)\|_{\mathbb{R}^{N}} d t \\
& +\int_{0}^{T}\left\|\partial_{5} L\left[x_{n}-x\right]_{\tau_{1}}^{\tau_{2}}(t)\right\|\left\|\dot{v}\left(t-\tau_{2}\right)\right\|_{\mathbb{R}^{N}} d t \\
= & \mathscr{I}_{2}+\mathscr{I}_{3}+\mathscr{I}_{4}+\mathscr{I}_{5},
\end{aligned}
$$

where

$$
\begin{aligned}
& \mathscr{I}_{2} \leq\left\|\partial_{2} L\left[x_{n}-x\right]_{\tau_{1}}^{\tau_{2}}(\cdot)\right\|\|v(t)\|_{L^{2}}, \\
& \mathscr{I}_{3} \leq\left\|\partial_{3} L\left[x_{n}-x\right]_{\tau_{1}}^{\tau_{2}}(\cdot)\right\|\|v(t)\|_{L^{2}\left(\left[-\tau_{1}, T\right], \mathbb{R}^{N}\right)}, \\
& \mathscr{I}_{4} \leq\left\|\partial_{4} L\left[x_{n}-x\right]_{\tau_{1}}^{\tau_{2}}(\cdot)\right\|\|\dot{v}(t)\|_{L^{2}}, \\
& \mathscr{I}_{5} \leq\left\|\partial_{3} L\left[x_{n}-x\right]_{\tau_{1}}^{\tau_{2}}(\cdot)\right\|\|\dot{v}(t)\|_{L^{2}\left(\left[-\tau_{1}, T\right], \mathbb{R}^{N}\right)} .
\end{aligned}
$$

On the other hand, $x_{n}(\cdot) \rightarrow x(\cdot)$ in $\mathscr{H}$. From Lebesgue's theorem, there exists $\mathbb{k}_{1}, \mathbb{k}_{2}, \mathbb{k}_{3} \subset \mathbb{N}$ such that $\mathbb{k}_{1} \subset \mathbb{k}_{2} \subset \mathbb{k}_{3}$ and

$$
\begin{array}{ll}
x_{k}(t) \longrightarrow x(t), & \text { a.e. } t \in[0, T], \quad \forall k \in \mathbb{k}_{1}, \\
\dot{x}_{k}(t) \longrightarrow \dot{x}(t), & \text { a.e. } t \in[0, T], \quad \forall k \in \mathbb{k}_{1}, \\
x_{k}(t) \longrightarrow x(t), & \text { a.e. } t \in\left[-\tau_{2}, 0\right], \quad \forall k \in \mathbb{k}_{2}, \\
\dot{x}_{k}(t) \longrightarrow \dot{x}(t), & \text { a.e. } t \in\left[-\tau_{2}, 0\right], \quad \forall k \in \mathbb{k}_{2}, \\
x_{k}(t) \longrightarrow x(t), & \text { a.e. } t \in\left[-\tau_{1},-\tau_{2}\right], \quad \forall k \in \mathbb{k}_{3} .
\end{array}
$$

Hence,

$$
\begin{array}{ll}
\dot{x}_{k}\left(t-\tau_{2}\right) \longrightarrow \dot{x}\left(t-\tau_{2}\right), & \text { a.e. } t \in\left[0, \tau_{2}\right], \quad \forall k \in \mathbb{k}_{2}, \\
x_{k}\left(t-\tau_{1}\right) \longrightarrow x\left(t-\tau_{1}\right), & \text { a.e. } t \in\left[0, \tau_{1}\right], \quad \forall k \in \mathbb{k}_{3} .
\end{array}
$$

Since $L(t, \cdot, \cdot, \cdot)$ is $C^{1}$-Carathéodory, assumption $\left(A_{2}\right)$ assures from Lebesgue's theorem that

$$
\left\|\partial_{i} L\left[x_{n}-x\right]_{\tau_{1}}^{\tau_{2}}(\cdot)\right\| \longrightarrow 0, \quad i=2,3,4,5 .
$$

This implies that $\mathscr{I}_{2}+\mathscr{I}_{3}+\mathscr{I}_{4}+\mathscr{I}_{5} \rightarrow 0$. Then, $J^{\prime}\left(x_{k}(\cdot)\right) \rightarrow$ $J^{\prime}(x(\cdot))$. The proof is complete.

Theorem 4 (necessary optimality conditions of Euler-Lagrange type for problem (1) and (2)). Under conditions $\left(A_{1}\right)$ and $\left(A_{2}\right)$, if $\bar{x}(\cdot)$ is a minimizer to problem (1) and (2), then $\bar{x}(\cdot)$ satisfies the following Euler-Lagrange equations with time delay:

$$
\begin{gathered}
\frac{d}{d t}\left\{\partial_{4} L[\bar{x}]_{\tau_{1}}^{\tau_{2}}(t)+\partial_{5} L[\bar{x}]_{\tau_{1}}^{\tau_{2}}\left(t+\tau_{2}\right)\right\} \\
=\partial_{2} L[\bar{x}]_{\tau_{1}}^{\tau_{2}}(t)+\partial_{3} L[\bar{x}]_{\tau_{1}}^{\tau_{2}}\left(t+\tau_{1}\right), \\
\text { a.e. } t \in\left[0, \top-\tau_{1}\right], \\
\frac{d}{d t}\left\{\partial_{4} L[\bar{x}]_{\tau_{1}}^{\tau_{2}}(t)+\partial_{5} L[\bar{x}]_{\tau_{1}}^{\tau_{2}}\left(t+\tau_{2}\right)\right\}=\partial_{2} L[\bar{x}]_{\tau_{1}}^{\tau_{2}}(t), \\
\text { a.e. } \left.t \in] \top-\tau_{1}, \top-\tau_{2}\right], \\
\left.\left.\frac{d}{d t} \partial_{4} L[\bar{x}]_{\tau_{1}}^{\tau_{2}}(t)=\partial_{2} L[\bar{x}]_{\tau_{1}}^{\tau_{2}}(t), \quad \text { a.e. } t \in\right] \top-\tau_{2}, \top\right] .
\end{gathered}
$$

Proof. If $\bar{x}(\cdot)$ is a minimizer to problem (1) and (2), then

$$
J^{\prime}(\bar{x}(\cdot))(v(\cdot))=0
$$

for all $v(\cdot) \in T(D, \bar{x}(\cdot))$; that is,

$$
\begin{aligned}
& \int_{0}^{\top}\left(\left\langle p_{2}(t), v(t)\right\rangle+\left\langle p_{3}(t), v\left(t-\tau_{1}\right)\right\rangle\right. \\
& \left.\quad+\left\langle p_{4}(t), \dot{v}(t)\right\rangle+\left\langle p_{5}(t), \dot{v}\left(t-\tau_{2}\right)\right\rangle\right) d t=0
\end{aligned}
$$

for all $v(\cdot) \in T(D, \bar{x}(\cdot))$ with $p_{i}(t)=\partial_{i} L[\bar{x}]_{\tau_{1}}^{\tau_{2}}(t), i=2,3,4,5$. Integration by parts yields

$$
\begin{aligned}
\int_{0}^{T}\left\langle p_{4}(t), \dot{v}(t)\right\rangle d t= & -\int_{0}^{T}\left\langle\dot{p}_{4}(t), v(t)\right\rangle d t, \\
\int_{0}^{T}\left\langle p_{5}(t), v\left(t-\tau_{2}\right)\right\rangle d t= & \left\langle p_{5}(T), v\left(T-\tau_{2}\right)\right\rangle \\
& +\int_{0}^{T}\left\langle\dot{p}_{5}(\tau), v\left(\tau-\tau_{2}\right)\right\rangle d \tau .
\end{aligned}
$$

By (31) and (32), we obtain that

$$
\begin{aligned}
\int_{0}^{T} & \left(\left\langle p_{2}(t), v(t)\right\rangle+\left\langle p_{3}(t), v\left(t-\tau_{1}\right)\right\rangle\right. \\
& \left.-\left\langle\dot{p}_{4}(t), v(t)\right\rangle+\left\langle\dot{p}_{5}(t), v\left(t-\tau_{2}\right)\right\rangle\right) d t \\
+ & \left\langle p_{5}(t), v\left(T-\tau_{2}\right)\right\rangle=0
\end{aligned}
$$


for all $v(\cdot) \in T(D, \bar{x}(\cdot))$. On the other hand,

$$
\begin{aligned}
\int_{0}^{T}\left\langle p_{3}(t), v\left(t-\tau_{1}\right)\right\rangle d t= & \int_{-\tau_{1}}^{T-\tau_{1}}\left\langle p_{3}\left(\tau+\tau_{1}\right), v(\tau)\right\rangle d \tau \\
= & \int_{-\tau_{1}}^{0}\left\langle p_{3}\left(\tau+\tau_{1}\right), v(\tau)\right\rangle d \tau \\
& +\int_{0}^{T-\tau_{1}}\left\langle p_{3}\left(\tau+\tau_{1}\right), v(\tau)\right\rangle d \tau \\
= & \int_{0}^{T-\tau_{1}}\left\langle p_{3}\left(\tau+\tau_{1}\right), v(\tau)\right\rangle d \tau, \\
\int_{0}^{T}\left\langle\dot{p}_{5}(t), v\left(t-\tau_{2}\right)\right\rangle d t= & \int_{-\tau_{2}}^{T-\tau_{2}}\left\langle\dot{p}_{5}\left(\tau+\tau_{2}\right), v(\tau)\right\rangle d \tau \\
= & \int_{-\tau_{2}}^{0}\left\langle\dot{p}_{5}\left(\tau+\tau_{2}\right) v(\tau)\right\rangle d \tau \\
& +\int_{0}^{T-\tau_{2}}\left\langle\dot{p}_{5}\left(\tau+\tau_{2}\right), v(\tau)\right\rangle d \tau \\
= & \int_{0}^{T-\tau_{2}}\left\langle\dot{p}_{5}\left(\tau+\tau_{2}\right), v(\tau)\right\rangle d \tau .
\end{aligned}
$$

Hence,

$$
\begin{aligned}
\int_{0}^{T}\left\langle p_{2}(t)-\dot{p}_{4}(t), v(t)\right\rangle d t \\
\quad+\int_{0}^{T-\tau_{1}}\left\langle p_{3}\left(t+\tau_{1}\right), v(t)\right\rangle d t \\
\quad-\int_{0}^{T-\tau_{2}}\left\langle\dot{p}_{5}\left(t+\tau_{2}\right), v(t)\right\rangle d t \\
\quad+\left\langle p_{5}(T), v\left(T-\tau_{2}\right)\right\rangle=0
\end{aligned}
$$

for all $v(\cdot) \in T(D, \bar{x}(\cdot))$. Put

$$
\begin{aligned}
& \bar{p}_{3}\left(t+\tau_{1}\right)= \begin{cases}p_{3}\left(t+\tau_{1}\right) & \text { if } t \in\left[0, T-\tau_{1}\right], \\
0 & \text { if } \left.t \in] T-\tau_{1}, T\right],\end{cases} \\
& q\left(t+\tau_{2}\right)= \begin{cases}\dot{p}_{5}\left(t+\tau_{2}\right) & \text { if } t \in\left[0, T-\tau_{2}\right], \\
0 & \text { if } \left.t \in] \top-\tau_{2}, \top\right]\end{cases}
\end{aligned}
$$

Then,

$$
\begin{gathered}
\int_{0}^{T}\left\langle p_{2}(t)-\dot{p}_{4}(t)+\bar{p}_{3}\left(t+\tau_{1}\right)-q\left(t+\tau_{2}\right), v(t)\right\rangle d t \\
+\left\langle p_{5}(T), v\left(T-\tau_{2}\right)\right\rangle=0
\end{gathered}
$$

for all $v(\cdot) \in T(D, \bar{x}(\cdot))$. In particular, for $v$ such that $v(\tau)=0$ for almost all $\tau \in\left[-\tau_{1}, 0\right]$ and $v(\tau)=0$ for almost all $\tau \in$ $\left[T-\tau_{2}, 0\right]$, we have

$$
p_{2}(t)-\dot{p}_{4}(t)+\bar{p}_{3}\left(t+\tau_{1}\right)-q\left(t+\tau_{2}\right)=0 \quad \text { a.e. } t \in[0, T]
$$

or

$$
\begin{gathered}
\dot{p}_{4}(t)+\dot{p}_{5}\left(t+\tau_{2}\right)=p_{2}(t)+p_{3}\left(t+\tau_{1}\right) \\
\text { a.e. } t \in\left[0, T-\tau_{1}\right], \\
\left.\left.\dot{p}_{4}(t)+\dot{p}_{5}\left(t+\tau_{2}\right)=p_{2}(t) \quad \text { a.e. } t \in\right] \top-\tau_{1}, \top-\tau_{2}\right], \\
\left.\left.\dot{p}_{4}(t)=p_{2}(t) \quad \text { a.e. } t \in\right] \top-\tau_{2}, \top\right] .
\end{gathered}
$$

The proof is complete.

\section{Optimal Control with Time Delays}

Now we prove existence of an optimal solution to more general problems of optimal control with time delays. The result is obtained via the exterior penalty method $[12,13]$ and Theorem 4 . The optimal control problem with time delays is defined as follows:

$$
\min \int_{0}^{T} l\left(t, x(t), \dot{x}\left(t-\tau_{2}\right), u(t)\right) d t
$$

subject to

$$
\begin{gathered}
\dot{x}(t)=A x\left(t-\tau_{1}\right)+B u(t), \quad t \in[0, T]=: I, \\
x(t)=\theta_{1}(t), \quad t \in\left[-\tau_{1},-\tau_{2}\right]=: I_{1}, \\
x(t)=\theta_{2}(t), \quad t \in\left[-\tau_{2}, 0\right]=: I_{2}, \\
x(T)=\alpha,
\end{gathered}
$$

where $x(\cdot) \in \mathscr{H}, u(\cdot) \in U_{0}=\left\{u(\cdot) \in L^{2}([0, \top], U): u(0)=0\right\}$, $A$ is an $N \times N$ matrix, $B$ is an $N \times m$ matrix, and $l:[0, \top] \times$ $\mathbb{R}^{N} \times \mathbb{R}^{N} \times \mathbb{R}^{m} \rightarrow \mathbb{R},(t, a, \bar{b}, c) \rightarrow l(t, a, \bar{b}, c)$. The final time $T>0$ is fixed in $\mathbb{R}, \tau_{1}$ and $\tau_{2}$ are two given positive real numbers such that $\tau_{2}<\tau_{1}<\mathrm{T}$, and, as before, $\theta_{1}(\cdot)$ and $\theta_{2}(\cdot)$ are given piecewise smooth functions. In the sequel, we denote by $\theta(\cdot)$ the function defined by $\theta(t)=\theta_{1}(t), t \in I_{1}$, and $\theta(t)=\theta_{2}(t), t \in I_{2}$. We make the following assumptions on the data of the problem.

$\left(H_{1}\right)$ The mapping $l$ is a $C^{1}$-Carathéodory mapping; that is, $l$ is $C^{1}$ in $(a, \bar{b}, c)$ for almost all $t \in[0, T]$ and is measurable in $t$ for every $(a, \bar{b}, c) \in \mathbb{R}^{N} \times \mathbb{R}^{N} \times \mathbb{R}^{m}$.

$\left(H_{2}\right)$ There exist $\gamma_{i}(\cdot) \in L^{2}\left(I, \mathbb{R}^{+}\right), i=1, \ldots, 5$, such that

$$
\begin{array}{r}
|l(t, a, \bar{b}, c)| \leq \gamma_{1}(t), \\
\left\|\partial_{i} l(t, a, \bar{b}, c)\right\| \leq \gamma_{i}(t) \\
\text { a.e. } t \in I, \quad i=2, \ldots, 4,
\end{array}
$$

where $\partial_{i} l$ is the partial derivative of $l$ with respect to its $i$ th argument, $i=2, \ldots, 4$.

$\left(H_{3}\right)$ There exists $\rho>0$ such that for almost all $t \in[0, T]$ and for all $(a, \bar{b}, c) \in \mathbb{R}^{N} \times \mathbb{R}^{N} \times \mathbb{R}^{m}$

$$
l(t, a, \bar{b}, c) \geq \rho\|c\|_{\mathbb{R}^{m}}
$$

$\left(H_{4}\right) l(t, a, \bar{b}, c)$ is convex in $(\bar{b}, c)$. 
Using the exterior penalty function method, we consider the following sequence of unconstrained optimal control problems corresponding to (40) and (41):

$$
\begin{gathered}
\inf \int_{0}^{\top} l\left(t, x(t), \dot{x}\left(t-\tau_{2}\right), u(t)\right) d t \\
+\frac{c_{n}}{2} \int_{0}^{\top}\left\|\dot{x}(t)-A x\left(t-\tau_{1}\right)-B u(t)\right\|_{\mathbb{R}^{N}}^{2} d t \\
x(t)=\theta_{1}(t) \quad \text { a.e. } t \in I_{1}, \\
x(t)=\theta_{2}(t) \quad \text { a.e. } t \in I_{2}, \\
x(\top)=\alpha, \\
x(\cdot) \in \mathscr{H}, \quad u(\cdot) \in U_{0},
\end{gathered}
$$

where $c_{n+1} \geq c_{n}, c_{n} \rightarrow \infty$. Denote

$$
\begin{gathered}
L_{n}(t, a, \bar{a}, b, \bar{b}, c):=l(t, a, \bar{b}, c)+\frac{c_{n}}{2}\|b-A \bar{a}+B c\|_{\mathbb{R}^{N}}^{2}, \\
J_{n}(x(\cdot), u(\cdot)) \\
:=\int_{0}^{\top} L_{n}\left(t, x(t), x\left(t-\tau_{1}\right), \dot{x}(t), \dot{x}\left(t-\tau_{2}\right), u(t)\right) d t, \\
D:=\{x(\cdot) \in \mathscr{H}: x(\top)=\alpha\} .
\end{gathered}
$$

The sequence of unconstrained optimal control problems takes then the following form:

$$
\begin{gathered}
\inf J_{n}(x(\cdot), u(\cdot)), \\
x(\cdot) \in D, \\
u(\cdot) \in U_{0},
\end{gathered}
$$

$n \in \mathbb{N}$

Lemma 5. The cone of tangents $T\left(U_{0}, u(\cdot)\right)$ is given by

$$
T\left(U_{0}, u(\cdot)\right)=\left\{w(\cdot) \in L^{2}\left(I, \mathbb{R}^{m}\right): w(0)=0\right\} .
$$

Proof. It is similar to the proof of Lemma 2.

It is well known that the penalty function method is a very effective technique for solving constrained optimization problems via unconstrained ones. The main question is the convergence of the sequence of solutions of the unconstrained optimal control problems to the original/constrained problem. Before giving the convergence theorem, we begin with some preparatory results, which are a direct consequence of the necessary optimality conditions given by Theorem 4.

Proposition 6. For every $n$, if $\left(x_{n}(\cdot), u_{n}(\cdot)\right) \in D \times U_{0}$ is an optimal solution to $\left(\mathscr{P}_{n}\right)$, then (i)

$$
\begin{gathered}
\frac{d}{d t} \phi_{n}(t)=A^{*} \phi_{n}\left(t+\tau_{1}\right)+\frac{1}{c_{n}} a_{n}(t)-\frac{1}{c_{n}} e_{n}(t) \\
\text { a.e. } t \in\left[0, \top-\tau_{1}\right], \\
\left.\left.\frac{d}{d t} \phi_{n}(t)=\frac{1}{c_{n}} a_{n}(t)-\frac{1}{c_{n}} e_{n}(t) \quad \text { a.e. } t \in\right] \top-\tau_{1}, \top-\tau_{2}\right], \\
\left.\left.\frac{d}{d t} \phi_{n}(t)=\frac{1}{c_{n}} a_{n}(t) \quad \text { a.e. } t \in\right] \top-\tau_{2}, \top\right], \\
B^{*} \phi_{n}(t)=\frac{1}{c_{n}} b_{n}(t) \quad \text { a.e. } t \in[0, \top],
\end{gathered}
$$

where

$$
\begin{aligned}
& \phi_{n}(t)=\dot{x}_{n}(t)-A x_{n}\left(t-\tau_{1}\right)+B u_{n}(t), \\
& a_{n}(t)=l_{a}^{\prime}\left(t, x_{n}(t), \dot{x}_{n}\left(t-\tau_{2}\right), u_{n}(t)\right), \\
& e_{n}(t)=l_{\bar{b}}^{\prime}\left(t, x_{n}(t), \dot{x}_{n}\left(t-\tau_{2}\right), u_{n}(t)\right), \\
& b_{n}(t)=l_{c}^{\prime}\left(t, x_{n}(t), \dot{x}_{n}\left(t-\tau_{2}\right), u_{n}(t)\right) ;
\end{aligned}
$$

(ii) there exists $M>0$ such that $\left\|\phi_{n}(t)\right\|_{X} \leq M$ for all $t \epsilon$ $[0, \top]$ and all $n$ sufficiently large.

Proof. (i) Let $\left(x_{n}(\cdot), u_{n}(\cdot)\right) \in D \times U_{0}$ be an optimal solution to $\left(\mathscr{P}_{n}\right)$. Then, by Lemmas 2 and 5 and Theorem 4 , we obtain the necessary conditions of item (i) for problem $\left(\mathscr{P}_{n}\right)$.

(ii) Since $u_{n}(0)=0$ and $\dot{\theta}\left(0^{+}\right)$exists, $\dot{x}_{n}(0)$ is defined and there exists $k>0$ such that $\left\|\phi_{n}(0)\right\| \leq k$. By the first equation of item (i), we have

$$
\begin{array}{r}
\phi_{n}(t)=\phi_{n}(0)+A^{*} \int_{0}^{t} \phi_{n}\left(\tau+\tau_{1}\right) d \tau \\
+\frac{1}{c_{n}} \int_{0}^{t}\left(a_{n}(\tau)+e_{n}(\tau)\right) d \tau, \\
t \in\left[0, \top-\tau_{1}\right] .
\end{array}
$$

Consequently,

$$
\begin{aligned}
\left\|\phi_{n}(t)\right\| & \leq\left\|\phi_{n}(0)\right\|+\left\|A^{*}\right\| \int_{0}^{t}\left\|\phi_{n}\left(\tau+\tau_{1}\right)\right\| d \tau+R_{n} \\
& \leq k+\alpha \int_{0}^{t}\left\|\phi_{n}\left(\tau+\tau_{1}\right)\right\| d \tau+R_{n}
\end{aligned}
$$

for all $t \in\left[0, \top-\tau_{1}\right]$ with $k=\left\|\phi_{n}(0)\right\|, \alpha=\left\|A^{*}\right\|$, and $R_{n}=$ $\left(1 / c_{n}\right)\left(\left\|\gamma_{2}(\cdot)\right\|_{L^{2}}+\left\|\gamma_{3}(\cdot)\right\|_{L^{2}}\right)$. By Gronwall's lemma, we obtain that

$$
\left\|\phi_{n}(t)\right\| \leq\left(k+R_{n}\right) \exp \left(\alpha\left(\top-\tau_{1}\right)\right) \quad \forall t \in\left[0, \top-\tau_{1}\right] .
$$


The second and third equalities of item (i) give

$$
\left.\left.\left\|\phi_{n}(t)\right\| \leq\left\|\phi_{n}\left(\top-\tau_{1}\right)\right\|+R_{n} \quad \forall t \in\right] \top-\tau_{1}, \top\right] .
$$

Now, inequalities (50) and (51) imply that

$$
\left\|\phi_{n}(t)\right\| \leq M_{n} \quad \forall n, \forall t \in[0, \top]
$$

with

$$
M_{n}=k \exp \left(\alpha\left(\top-\tau_{1}\right)\right)+R_{n}\left(1+\exp \left(\alpha\left(\top-\tau_{1}\right)\right)\right) .
$$

Since $R_{n} \rightarrow 0$, there exists $M>0$ such that

$$
\left\|\phi_{n}(t)\right\|_{\mathbb{R}^{N}} \leq M
$$

for all $t \in[0, \top]$ and for all $n$ large.

We are now ready to prove the convergence theorem, which reads as follows.

Theorem 7 (penalty convergence theorem). If hypotheses $\left(H_{1}\right)-\left(H_{4}\right)$ hold and problem (40) and (41) has a finite value, then the sequence $\left(x_{n}(\cdot), u_{n}(\cdot)\right)_{n}$ of solutions to $\left(\mathscr{P}_{n}\right)$ contains a subsequence $\left(x_{k}(\cdot), u_{k}(\cdot)\right)_{k}$ such that

(i) $x_{k}(\cdot) \rightarrow x(\cdot)$ strongly in $C\left(I, \mathbb{R}^{N}\right)$;

(ii) $u_{k}(\cdot) \rightarrow u(\cdot)$ weakly in $L^{2}\left(I, \mathbb{R}^{m}\right)$;

(iii) $\dot{x}_{k}(\cdot) \rightarrow \dot{x}(\cdot)$ weakly in $L^{2}\left(I, \mathbb{R}^{N}\right)$;

with $(x(\cdot), u(\cdot))$ a solution to problem (40) and (41).

Proof. Let $\left(x_{n}(\cdot), u_{n}(\cdot)\right) \in D \times U_{0}$ be an optimal solution to $\left(\mathscr{P}_{n}\right)$ for every $n$. By Proposition 6,

$$
\begin{aligned}
\left\|\dot{x}_{n}(t)\right\| & \leq M+\|A\|\left\|x_{n}\left(t-\tau_{1}\right)\right\|+\|B\|\left\|u_{n}(t)\right\| \\
& \leq M+\beta\left\|x_{n}\left(t-\tau_{1}\right)\right\|+\sigma\left\|u_{n}(t)\right\| .
\end{aligned}
$$

Because

$$
\begin{gathered}
x_{n}(t)=\theta(0)+\int_{0}^{t} \dot{x}_{n}(\tau) d \tau \quad \forall t \in[0, \top], \\
x_{n}(t)=\theta(t) \quad \text { a.e. } t \in\left[-\tau_{1}, 0\right]
\end{gathered}
$$

it follows that

$$
\begin{aligned}
\left\|x_{n}(t)\right\| \leq & \|\theta(0)\|+\int_{0}^{t}\left\|\dot{x}_{n}(\tau)\right\| d \tau \\
\leq & \|\theta(0)\|+M \top+\beta \int_{0}^{t}\left\|x_{n}(\tau-h)\right\| d \tau \\
& +\sigma \int_{0}^{t}\left\|u_{n}(\tau)\right\| d \tau .
\end{aligned}
$$

On the other hand, if $\mathscr{M}$ denotes the finite value of (40) and (41), then

$$
\int_{0}^{\top} l\left(t, x_{n}(t), u_{n}(t)\right) d t \leq J_{n}\left(x_{n}(\cdot), u_{n}(\cdot)\right) \leq \mathscr{M}
$$

By assumption $\left(H_{3}\right)$, there exists $K>0$ such that

$$
\left\|u_{n}(\cdot)\right\|_{L^{2}} \leq K
$$

Thus,

$$
\begin{aligned}
\left\|x_{n}(t)\right\| \leq & \|\theta(0)\|+M \top+\sigma \top K \\
& +\beta \int_{-\tau_{1}}^{0}\|\theta(\tau)\| d \tau+\beta \int_{0}^{t-\tau_{1}}\left\|x_{n}(\tau)\right\| d \tau .
\end{aligned}
$$

By Gronwall's lemma, we obtain that

$$
\left\|x_{n}(t)\right\| \leq \psi \text { for } n \text { sufficiently large, } \forall t \in[0, \top],
$$

where

$$
\psi=\left(\|\theta(0)\|+M \top+\sigma \top K+\beta \tau_{1}\|\theta(\cdot)\|\right) \exp \left(\beta\left(\top-\tau_{1}\right)\right) .
$$

Similarly, for $n$ sufficiently large,

$$
\left\|\dot{x}_{n}(t)\right\| \leq M+\beta\left\|x_{n}\left(t-\tau_{1}\right)\right\|+\sigma\left\|u_{n}(t)\right\| .
$$

For all $t \in[0, h]$, we have

$$
\left\|\dot{x}_{n}(t)\right\| \leq M+\gamma(t)+\beta\left\|\theta\left(t-\tau_{1}\right)\right\|+\sigma\left\|u_{n}(t)\right\|=\omega(t) .
$$

Since $\omega(\cdot) \in L^{2}(I, \mathbb{R})$ and $\left(u_{n}(\cdot)\right)_{n}$ is bounded in $L^{2}\left(I, \mathbb{R}^{m}\right)$, with $[0, h]$ of finite measure, there exists $\varrho>0$ such that

$$
\left\|\dot{x}_{n}(\cdot)\right\| \leq \varrho \quad \text { in } L^{2}\left(\left[0, \tau_{1}\right], \mathbb{R}^{N}\right) \text {, for } n \text { sufficiently large. }
$$

For all $t \in\left[\tau_{1}, \top\right]$ we have

$$
\left\|\dot{x}_{n}(t)\right\| \leq M+\gamma(t)+\beta \psi+\sigma\left\|u_{n}(t)\right\| .
$$

As before, we can assert that

$$
\begin{aligned}
& \exists \widehat{\varrho}>0:\left\|\dot{x}_{n}(\cdot)\right\| \leq \widehat{\varrho} \\
& \text { in } L^{2}\left(\left[\tau_{1}, \top\right], \mathbb{R}^{N}\right) \text { for } n \text { sufficiently large. }
\end{aligned}
$$

By (65) and (67), there exists $\eta>0$ such that

$$
\left\|\dot{x}_{n}(\cdot)\right\| \leq \eta
$$

in $L^{2}\left([0, \top], \mathbb{R}^{N}\right)$ for $n$ sufficiently large. Therefore, there exists a subsequence $\left(\dot{x}_{k}(\cdot)\right)_{k}$ of $\left(\dot{x}_{n}(\cdot)\right)_{n}$ converging to $\sigma(\cdot) \epsilon$ $L^{2}\left(I, \mathbb{R}^{N}\right)$. Since $x_{n}(t)=\theta(0)+\int_{0}^{t} \dot{x}_{n}(\tau) d \tau$ for all $t \in I$, by the use of $(61)$, the sequence $\left(x_{n}(\cdot)\right)_{n}$ is equi-bounded and equi-continuous (because $\left(\dot{x}_{n}(\cdot)\right)_{n}$ is bounded in $L^{2}\left(I, \mathbb{R}^{N}\right)$ ). Ascoli's theorem implies that

$$
x_{k}(\cdot) \longrightarrow x(\cdot) \quad \text { strongly in } C\left(I, \mathbb{R}^{N}\right) .
$$

Since

$$
x_{k}(0)=\theta(0), \quad \int_{0}^{t} \dot{x}_{n}(\tau) d \tau \longrightarrow \int_{0}^{t} \sigma(\tau) d \tau,
$$


we obtain that $x(t)=\theta(0)+\int_{0}^{t} \sigma(\tau) d \tau$ and $\dot{x}(t)=\sigma(t)$ a.e. $t \epsilon$ $I$. The sequence $\left(u_{n}(\cdot)\right)_{n}$ is bounded in $L^{2}\left(I, \mathbb{R}^{m}\right)$. Thus, there exists a subsequence $\left(u_{k}(\cdot)\right)_{k}$ such that $u_{k}(\cdot) \rightarrow u(\cdot)$ weakly in $L^{2}\left(I, \mathbb{R}^{N}\right)$. To complete the proof, we show that $(x(\cdot), u(\cdot))$ is an optimal solution to (40)-(41). By Proposition 6, we have

$$
B^{*} \phi_{k}(t)=\frac{1}{c_{k}} b_{k}(t) \quad \text { a.e. } t \in I .
$$

Hence,

$$
\int_{0}^{t}\left\|B^{*} \phi_{k}(\tau)\right\| d \tau=\frac{1}{c_{k}} \int_{0}^{t}\left\|b_{k}(\tau)\right\| d \tau \leq \frac{1}{c_{k}} M
$$

with $M=\mathrm{T}\left\|\gamma_{4}(\cdot)\right\|_{L^{2}}$. We conclude that

$$
\int_{0}^{t} B^{*} \phi_{k}(\tau) d \tau \longrightarrow 0 \quad \forall t \in I .
$$

On the other hand,

$$
\int_{0}^{t} B^{*} \phi_{k}(\tau) d \tau \longrightarrow \int_{0}^{t} B^{*} \phi(\tau) d \tau \quad \forall t \in I .
$$

Consequently,

$$
\int_{0}^{t} B^{*} \phi(\tau) d \tau=0 \quad \forall t \in I .
$$

This implies that

$$
B^{*} \phi(t)=0 \quad \forall t \in I .
$$

Thus,

$$
\begin{gathered}
\phi(t)=0 \quad \forall t \in I, \\
\dot{x}(t)=A x\left(t-\tau_{1}\right)+B u(t) \quad \forall t \in I
\end{gathered}
$$

and $x(t)=\theta(t)$ a.e. $t \in\left[-\tau_{1}, 0\right], x(T)=\alpha$. Then, $(x(\cdot), u(\cdot))$ is an admissible pair and

$$
\int_{I} l\left(t, x(t), \dot{x}\left(t-\tau_{2}\right), u(t)\right) d t \geq \mathscr{M}
$$

On the other hand,

$$
\int_{I} l\left(t, x_{k}(t), \dot{x}_{k}\left(t-\tau_{2}\right), u_{k}(t)\right) d t \leq \mathscr{M}
$$

Now the hypotheses $\left(H_{1}\right),\left(H_{2}\right)$, and $\left(H_{4}\right)$, together with Lebesgue's theorem, assert that

$$
\int_{I} l\left(t, x(t), \dot{x}\left(t-\tau_{2}\right), u(t)\right) d t \leq \mathscr{M}
$$

that is,

$$
\int_{I} l\left(t, x(t), \dot{x}\left(t-\tau_{2}\right), u(t)\right) d t=\mathscr{M}
$$

This implies that the pair $(x(\cdot), u(\cdot))$ is a solution to problem (40) and (41).

\section{Conclusion}

New optimality conditions for problems of the calculus of variations and optimal control with time delays, where the delay in the unknown function differs from the delay in its derivative/control, were obtained. The proofs are first given in the simpler context of the delayed calculus of variations and then extended to delayed optimal control problems by using a penalty method. New results include a convergence theorem (see Theorem 7), which is of great practical interest because it allows us to obtain a solution to a delayed optimal control problem by considering a sequence of simpler problems of the calculus of variations. Previous results in the literature $[2,3,11]$ consider the delay in the unknown function to be the same as the delay in its derivative. There is, however, no justification for the delays to be the same. In contrast with those results, here we consider the case of multiple time delays. Moreover, the procedure of our proofs is completely different from the case of one time delay only, which relies on the Lagrange multiplier method. Such approach introduces a new unknown function, the Lagrange multiplier, for which it is hard to set the interpolation space. Indeed, the Lagrange multiplier must be carefully selected in order to be possible to obtain an accurate solution. Otherwise, the resulting system of equations may become singular, in particular if the number of degrees of freedom is too large. Here we use a penalty method, which requires only the choice of one scalar parameter. Big values of this parameter are used in order to impose the boundary conditions in a proper manner. Furthermore, in our case the use of the penalty method replaces a constrained optimization problem (the delayed optimal control problem) by a sequence of unconstrained problems of the calculus of variations with time delay whose solutions converge to the solution of the original constrained problem. Similarly to [11], our results can be easily extended for controls with time delay.

\section{Conflict of Interests}

The authors declare that there is no conflict of interests regarding the publication of this paper.

\section{Acknowledgments}

This work was partially supported by Portuguese funds through the Center for Research and Development in Mathematics and Applications (CIDMA) and The Portuguese Foundation for Science and Technology (FCT), within Project PEst-OE/MAT/UI4106/2014. Torres was also supported by the FCT Project PTDC/EEI-AUT/1450/2012, cofinanced by FEDER under POFC-QREN with COMPETE reference FCOMP-01-0124-FEDER-028894. The authors are grateful to two anonymous referees for valuable remarks and comments, which significantly contributed to the quality of the paper.

\section{References}

[1] C. Dacka, "On the controllability of nonlinear systems with time-variable delays," IEEE Transactions on Automatic Control, vol. 26, no. 4, part 1, pp. 956-959, 1981. 
[2] G. S. F. Frederico, T. Odzijewicz, and D. F. M. Torres, "Noether's theorem for non-smooth extremals of variational problems with time delay," Applicable Analysis., vol. 93, no. 1, pp. 153-170, 2014.

[3] G. S. F. Frederico and D. F. M. Torres, "A nondifferentiable quantum variational embedding in presence of time delays," International Journal of Difference Equations, vol. 8, no. 1, pp. 49-62, 2013.

[4] J.-Q. Sun and Q. Ding, Advances in Analysis and Control of Time-Delayed Dynamical Systems, World Scientific Publishing, Singapore, 2013.

[5] A. Debbouche and D. F. M. Torres, "Approximate controllability of fractional nonlocal delay semilinear systems in Hilbert spaces," International Journal of Control, vol. 86, no. 9, pp. 15771585, 2013.

[6] A. Debbouche and D. F. M. Torres, "Approximate controllability of fractional delay dynamic inclusions with nonlocal control conditions," Applied Mathematics and Computation, vol. 243, pp. 161-175, 2014.

[7] J.-P. Richard, "Time-delay systems: an overview of some recent advances and open problems," Automatica, vol. 39, no. 10, pp. 1667-1694, 2003.

[8] D. Salamon, "On controllability and observability of time delay systems," IEEE Transactions on Automatic Control, vol. 29, no. 5, pp. 432-439, 1984

[9] L. Göllmann, D. Kern, and H. Maurer, “Optimal control problems with delays in state and control variables subject to mixed control-state constraints," Optimal Control Applications \& Methods, vol. 30, no. 4, pp. 341-365, 2009.

[10] L. Göllmann and H. Maurer, "Theory and applications of optimal control problems with multiple time-delays," Journal of Industrial and Management Optimization, vol. 10, no. 2, pp. 413441, 2014.

[11] G. S. F. Frederico and D. F. M. Torres, "Noether's symmetry theorem for variational and optimal control problems with time delay," Numerical Algebra, Control and Optimization, vol. 2, no. 3, pp. 619-630, 2012.

[12] M. Benharrat and H. Mokhtar-Kharroubi, "Exterior penalty in optimal control problem with state-control constraints," Rendiconti del Circolo Matematico di Palermo, vol. 59, no. 3, pp. 389-403, 2010

[13] S. Y. Serovaiskii, "An approximate penalty method in the problem of the optimal control of nonsmooth singular systems," Mathematical Notes, vol. 76, no. 5-6, pp. 834-843, 2004. 


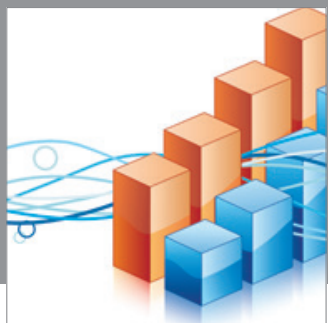

Advances in

Operations Research

mansans

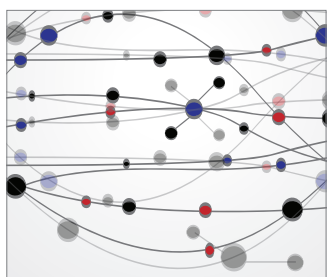

The Scientific World Journal
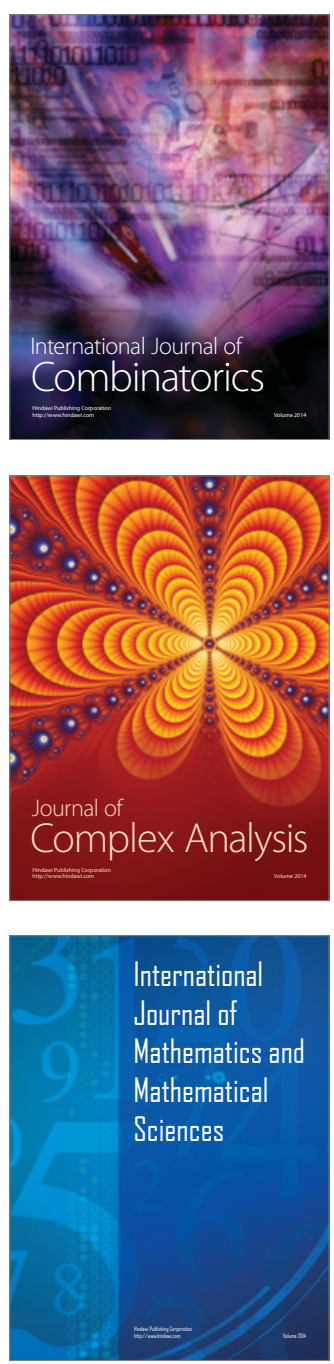
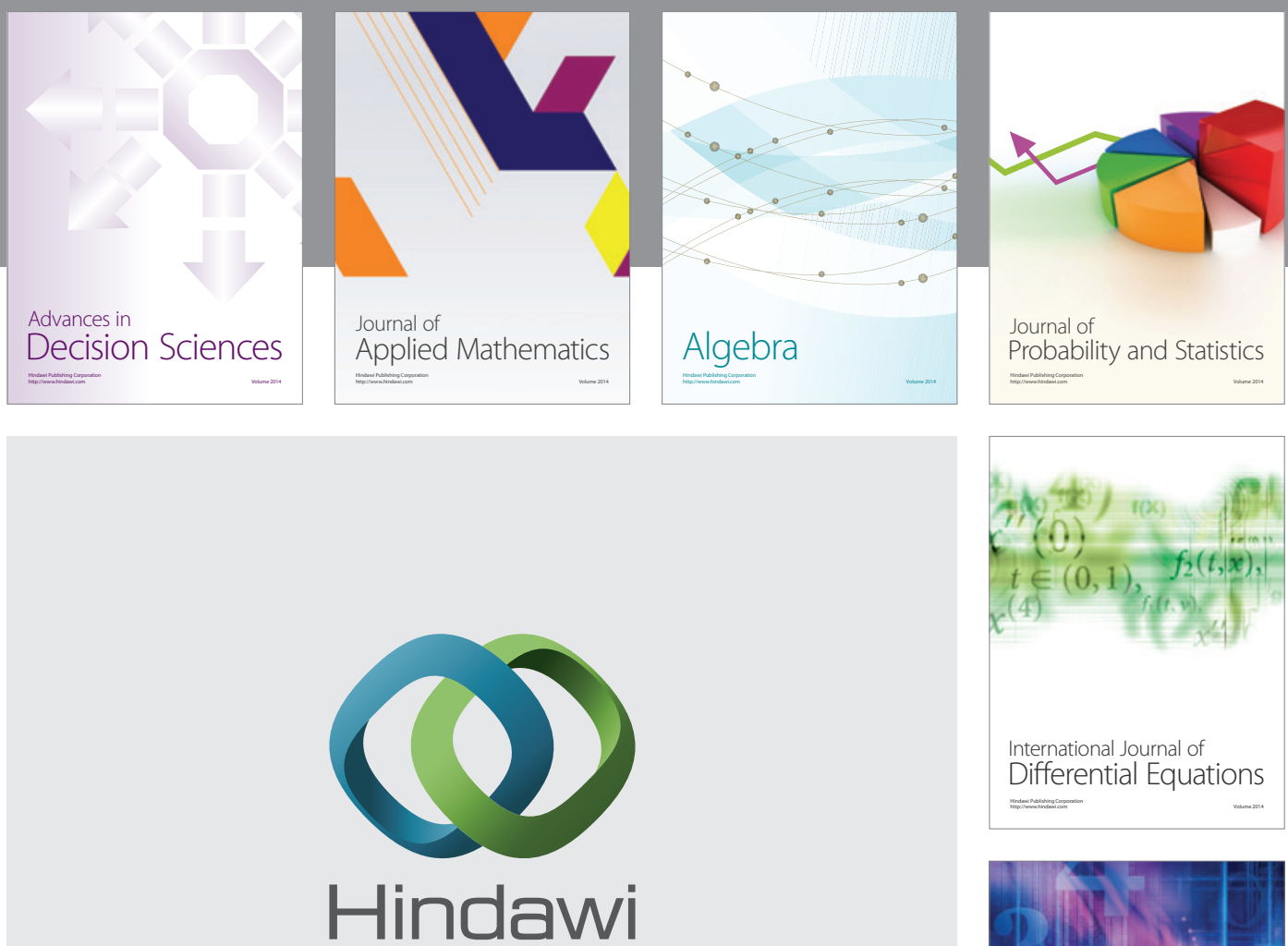

Submit your manuscripts at http://www.hindawi.com
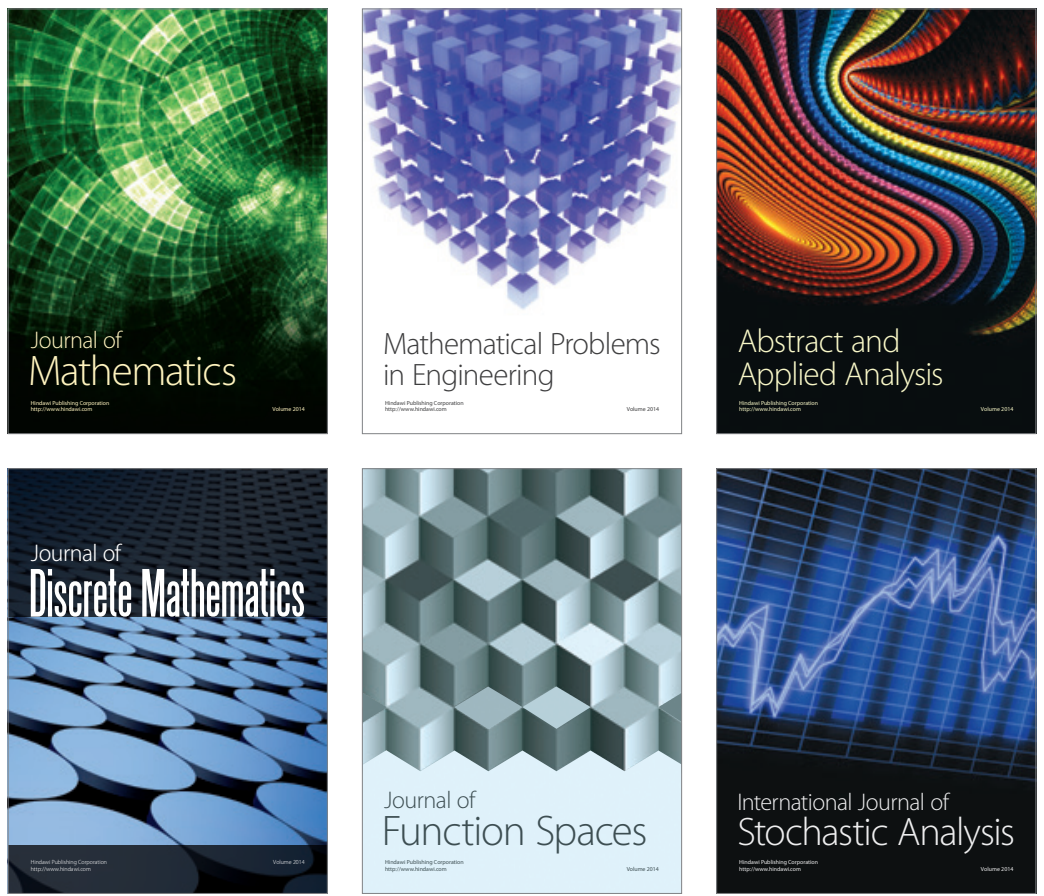

Journal of

Function Spaces

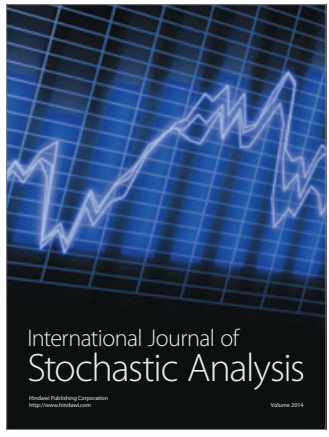

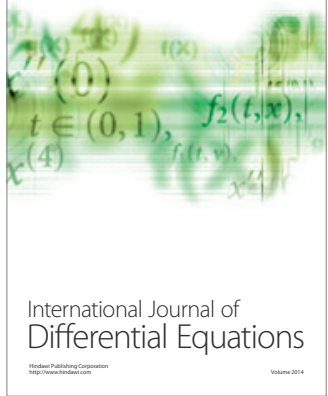
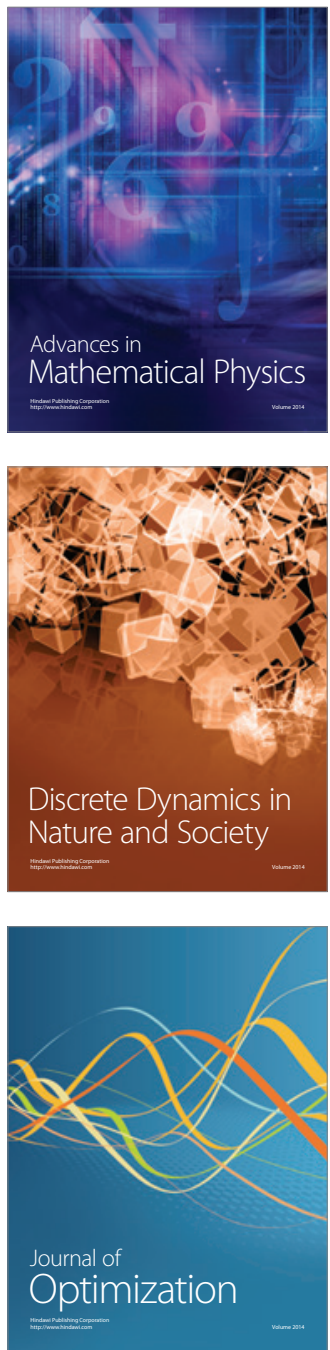